\title{
Recovering a tensor on the boundary from polarization and phase measurements
}

\author{
S Holman \\ Mathematics Department \\ University of Washington - Seattle \\ C138 Padelford, Box \# 354350 \\ Seattle, WA 98195 \\ USA \\ E-mail: holmansf@math. washington.edu
}

\begin{abstract}
We consider the problem of recovering a quasi-isotropic medium from polarization measurements made along the boundary. We show that the polarization and phase data uniquely determine a 2-tensor $f$ and all of its normal derivatives on the boundary in dimension $n \geq 4$. In dimension $n=3$ we establish a similar result which accounts for a natural non-uniqueness in the inverse problem.
\end{abstract}




\section{Introduction}

Suppose we are able to set the polarization of light rays entering a medium, and then measure the polarization as they exit. If the medium being studied is anisotropic then the polarization of the exiting light rays will be different from the entering rays, and with this information, the change in polarization, we would like to be able to say something about the interior of the medium. Specifically we would like to be able to obtain information about the dielectric permittivity. Indeed, we will assume that the medium is not magnetic, and that the dieletric permittivity in the interior is given by

$$
\epsilon_{l}^{j}(x)=\epsilon(x) \delta_{l}^{j}+\frac{1}{k} \chi_{l}^{j}(x) .
$$

Here $k$ is the wave number, and because of the presence of the factor $1 / k$ in front of the anisotropic part $\chi_{l}^{j}$ the zero approximation of geometric optics is the same as that for the isotropic medium $\epsilon_{l}^{j}=\epsilon(x) \delta_{l}^{j}$. Such a medium is called quasi-isotropic and was originally proposed for study by Kravstov [1]. Physical media with sufficiently weak anisotropies such as plasmas and weakly stressed elastic media may be well modeled by quasi-isotropic media (see [1]).

The inverse problem of recovering $\chi$, the anisotropic part of $\epsilon_{l}^{j}$, from polarization measurements of light with wave number $k$ on the boundary of a given region $\Omega \subset \mathbb{R}^{n}$ assuming that the isotropic background, $\epsilon(x)$, is known is studied in [2] and [3]. Through the method of geometric optics this problem is changed to the geometric problem on a Riemannian manifold described below, and it is this geometric problem that we consider here. For details of this conversion, and also further details of all the objects defined below, see [3]. The polarization vector $\eta$ introduced below corresponds physically to the zero approximation electrical field normalized to unit length in the background isotropic metric $\epsilon(x) \delta_{l j}$. It is measurements of this vector that we will consider here, although it should be noted that this vector actually contains both phase and polarization information. The same problem without this phase information is considered in [2] and [3]. Extensive work also exists on the problem of polarization tomography in the context of the photoelastic effect where inversion techniques are known as integrated photoelasticity ( [4], [5], [6], [7], [8]).

Let $(M, g)$ be a compact Riemannian manifold with nonempty, strictly convex boundary such that no geodesic has infinite length. Such a manifold will be called compact non-trapping (CNT). Let $(M, g)$ be a CNT manifold of dimension $n \geq 3$. Let $i: \partial M \rightarrow M$ be the inclusion mapping, and first consider $i^{*}\left(T M \bigoplus T^{\mathbb{C}} M\right)$, the direct sum bundle over $\partial M$. Define the two subsets of this bundle

$$
\Psi_{ \pm}(\partial M)=\left\{(\xi, \eta) \in i^{*}\left(T M \bigoplus T^{\mathbb{C}} M\right):\|\xi\|_{g}=1, \pm\langle\xi, \nu\rangle_{g} \geq 0\right\}
$$

where $\nu$ is the outward pointing normal vector to $\partial M$. Now, take any $f \in \tau_{1}^{1}(M)$ where $\tau_{1}^{1}(M)$ denotes the space of smooth sections of the complexified bundle of $(1,1)$ tensors over $M$. The problem we will consider is that of determining $f$ given $g$ and the map $\mathcal{F}[f]: \Psi_{-}(\partial M) \rightarrow \Psi_{+}(\partial M)$ defined as follows. For $\left(\xi, \eta_{0}\right) \in \Psi_{-}(\partial M)$ let $\gamma_{\xi}$ be the 
unit speed geodesic in $(M, g)$ with initial data $\xi$. Assume this geodesic has length $l$ so that $\gamma_{\xi}(l) \in \partial M$. Now consider the vector field $\eta$ along $\gamma_{\xi}$ that solves the initial value problem

$$
\frac{D \eta}{\mathrm{ds}}=\left(P_{\dot{\gamma}_{\xi}} f\right) \eta \quad, \quad \eta\left(\gamma_{\xi}(0)\right)=\eta_{0}
$$

Here $D \eta /$ ds is the covariant derivative along $\gamma_{\xi}$, and $P_{\dot{\gamma}_{\xi}} f=\pi_{\dot{\gamma}_{\xi}} f \pi_{\dot{\gamma}_{\xi}}$ where $\pi_{\dot{\gamma}_{\xi}}$ is the orthogonal projection onto the subspace of $T_{\gamma_{\xi}}^{\mathbb{C}} M$ perpendicular to $\dot{\gamma}_{\xi}$. Now we define $\mathcal{F}[f]\left(\xi, \eta_{0}\right)=\left(\dot{\gamma}_{\xi}(l), \eta\left(\gamma_{\xi}(l)\right)\right)$. In [2] uniqueness and stability of this inverse problem are established under curvature assumptions on $g$ and a priori smallness assumptions on $f$. An inverse problem corresponding to (1.1) without the projection $P_{\dot{\gamma}_{\xi}}$ is considered in [9]. When the dimension $n$ is 3 , this inverse problem corresponds to the physical problem described above of determining the anisotropic part of the dielectric permittivity, $\chi$, of a quasi-isotropic medium from polarization and phase data recorded around the boundary assuming that the isotropic part is known. In fact $\chi$ and $f$ are related by the formula

$$
f=\frac{i \chi}{2 \epsilon} \text {. }
$$

In this setting the geodesics are considered to be light rays, and the vector field $\eta$ along $\gamma_{\xi}$ given by (1.1) is the polarization vector mentioned above. We imagine that we can set the initial polarization vector, $\eta_{0}$, as any light ray enters the medium, and then measure the polarization vector, $\eta(l)$, as that ray leaves the medium. We note that to accomplish these measurements in practice requires both measuring the polarization ellipse of the exiting light ray, and the phases in two perpendicular directions.

We now reformulate the geometric inverse problem presented above. First we must introduce some notation. A semi-basic $(1,1)$ tensor field on $M$ is a tensor field $U$ on $T M$ whose representation in any natural coordinates $(x, \xi)$ is of the form

$$
U=u_{i}^{j}(x, \xi) \frac{\partial}{\partial \xi^{j}} \otimes d x^{i} .
$$

The bundle of complex $(1,1)$ semi-basic tensors over $T M$ is naturally isomorphic to the pullback bundle $\pi^{*}\left(T_{1}^{1} \mathbb{C} M\right)$ where $\pi: T M \rightarrow M$ is the projection mapping, and thus semi-basic tensors at a given point $(x, \xi)$ can be considered as linear maps on $T_{x}^{\mathbb{C}} M$. The set of semi-basic tensor fields restricted to the unit sphere bundle $\Omega M$ will be denoted $\beta_{1}^{1}(\Omega M)$. Given any tensor field $f \in \tau_{1}^{1}(M)$ consider the following equation for $U \in \beta_{1}^{1}(\Omega M)$ where $H$ is differentiation with respect to the geodesic flow.

$$
H U(\xi)=\left[\left(P_{\xi} f\right)(x)\right] U(\xi) \quad \text { on } \Omega M,\left.\quad U\right|_{\partial_{-} \Omega M}=E
$$

where $\partial_{-} \Omega M$ is the space of inward pointing unit vectors on $\partial M$. $U$ can be thought of as the "fundamental matrix" for (1.1). In fact problems (1.1) and (1.2) are related as follows.

$$
\eta\left(\gamma_{\xi}(t)\right)=U\left(\dot{\gamma}_{\xi}(t)\right) \mathcal{I}_{0, t}^{\gamma_{\xi}} \eta_{0}
$$

Here $\mathcal{I}_{0, t}^{\gamma_{\xi}}$ is parallel translation along $\gamma_{\xi}$. In the context of (1.2), the inverse problem is to determine $f$ from the metric $g$ and $\left.U\right|_{\partial_{+} \Omega M}$ (where $\partial_{+} \Omega M$ is the space of outward pointing vectors). We will call $\left.U\right|_{\partial_{+} \Omega M}$ the polarization data corresponding to $f$. 
The main result of this paper is the following theorem

Theorem 1. Let $(M, g)$ be a CNT manifold of dimension at least 4 and let $f \in \tau_{1}^{1}(M)$ be a smooth tensor field. Then in any coordinates all derivatives of all components of $f$ on $\partial M$ are uniquely determined by the polarization data of $f$.

As a corollary of this theorem we also immediately have the following.

Corollary 2. Suppose $f$ is as in theorem 1 and is also real-analytic. Then $f$ is the unique real-analytic tensor field giving rise to the same polarization data.

We will also establish a result similar to theorem 1 in the three dimensional case, but delay its statement until the end of section 2 . In section 2 we examine the natural non-uniqueness for this inverse problem which occurs in three dimensions, and give a statement of the result corresponding to theorem 1 in that case. Section 3 contains the main identity used to establish theorem 1. In Section 4 the proof of theorem 1 is given, and finally in section 5 the proof of the corresponding three dimensional result is given.

\section{Non-uniqueness in the three dimensional case}

In dimension $n=3$ there is a natural non-uniqueness to the inverse problem which we will now describe. This is a reformulated and slightly expanded version of what can be found in [2]. First we will introduce some notation and a few geometric objects. The space of smooth k-forms on $M$ will be denoted by $\Lambda^{k} T^{*} M$. When $(M, g)$ is oriented we may define the Hodge star operator $*: \Lambda^{k} T^{*} M \rightarrow \Lambda^{3-k} T^{*} M$ and also the coderivative $\delta=* d *$ where $d$ is the exterior derivative. The volume form for the metric $g$ will be written as $\mathrm{d} v_{g}$.

The non-uniqueness now results from the fact that, as we will see below, for any "coexact" tensor, $f \approx \delta h$, the polarization data is entirely determined by the values of the potential $h$ at the boundary. More precisely, we have the following theorem.

Theorem 3. If a CNT manifold $M^{3}$ is orientable and $h_{1}, h_{2} \in C^{\infty}(M)$ are such that $\left.h_{1}\right|_{\partial M}=\left.h_{2}\right|_{\partial M}$, then the polarization data for $f_{i}=\left(\delta\left(h_{i} \mathrm{~d} v_{g}\right)\right)^{\#}(i=1,2)$ are the same. The \# indicates that one of the indices of $\delta\left(h \mathrm{~d} v_{g}\right)$ has been raised.

Remark 1. The orientability hypothesis here arises because of the need to globally define $\delta$. However, it is possible to find a similar non-uniqueness result in the case where $M$ is not orientable by applying this theorem on an orientable submanifold of $M$ with boundary.

Proof. Let $\gamma$ be any unit speed geodesic in $M$ starting at $x \in \partial M$ with length $l$. We can then choose coordinates $\left\{x^{1}, x^{2}, x^{3}\right\}$ on a tubular neighborhood of $\gamma$ such that $\dot{\gamma}=\partial_{x^{3}}$, and $\left\{\partial_{x_{1}}, \partial_{x_{2}}, \partial_{x_{3}}\right\}$ is a parallel orthonormal frame along the entire length of $\gamma$. If $h_{i}$ is

as in the statement of the theorem for $i=1$ or 2 , then we can calculate $P_{\partial_{x_{3}}} f_{i}$ at points on $\gamma$ in these coordinates as follows. Letting $h=h_{1}$ or $h_{2}$ we have

$$
\begin{aligned}
\delta\left(h \mathrm{~d} v_{g}\right) & =* d(h)=*\left(\partial_{x^{1}} h d x^{1}+\partial_{x^{2}} h d x^{2}+\partial_{x^{3}} h d x^{3}\right) \\
& =\partial_{x^{1}} h d x^{2} \wedge d x^{3}-\partial_{x^{2}} h d x^{1} \wedge d x^{3}+\partial_{x^{3}} h d x^{1} \wedge d x^{2} .
\end{aligned}
$$


Thus at points on $\gamma$

$$
\left[P_{\dot{\gamma}}\left(\delta\left(h \mathrm{~d} v_{g}\right)\right)^{\#}\right]=\partial_{x^{3}} h\left(\partial_{x^{1}} \otimes d x^{2}-\partial_{x^{2}} \otimes d x^{1}\right) .
$$

Now take any $\eta_{0} \in T_{\gamma(0)}^{\mathbb{C}} M$ as an initial vector. We must solve the following system of equations along $\gamma$

$$
\frac{\partial \eta}{\partial t}=\partial_{x^{3}} h \partial_{x^{1}} \wedge d x^{2}(\eta) \quad, \quad \eta(0)=\eta_{0}
$$

It is not hard to see that the solution to this system is given by

$$
\eta(\gamma(t))=\left(\begin{array}{c}
\cos (h(\gamma(t))-h(x)) \eta_{0}^{1}+\sin (h(\gamma(t))-h(x)) \eta_{0}^{2} \\
\cos (h(\gamma(t))-h(x)) \eta_{0}^{2}-\sin (h(\gamma(t))-h(x)) \eta_{0}^{1} \\
\eta_{0}^{3}
\end{array}\right)
$$

Finally, let $\gamma(l)=y$ and note that $x, y \in \partial M$. Then we have

$$
\eta(y)=\left(\begin{array}{c}
\cos (h(y)-h(x)) \eta_{0}^{1}+\sin (h(y)-h(x)) \eta_{0}^{2} \\
\cos (h(y)-h(x)) \eta_{0}^{2}-\sin (h(y)-h(x)) \eta_{0}^{1} \\
\eta_{0}^{3}
\end{array}\right)
$$

Now we can see that since $\left.h_{1}\right|_{\partial M}=\left.h_{2}\right|_{\partial M}$, in fact the polarization data for $f_{1}$ and $f_{2}$ as defined in the statement of the theorem are the same.

In view of the previous theorem we make the following definition for notational convenience. A tensor field $f \in \tau_{1}^{1}(M)$ will be called coexact if $f=\left(\delta\left(h \mathrm{~d} v_{g}\right)\right)^{\#}$ for some $h \in C^{\infty}(M)$. We also define the tangential component of $f$ to be $\mathbf{t} f=i^{*}(f)_{b}$ and the normal component of $f$ to be $\mathbf{n} f=(f)_{b}-\mathbf{t} f$. The $b$ symbol indicates that an index is lowered.

Now, having reviewed the non-uniqueness we state the result corresponding to theorem 1 for the 3 dimensional case. Since the tensors which, according to theorem 3 , produce non-unique polarization data are precisely the coexact tensors, it is natural to consider the uniqueness problem only on a subspace of $\tau_{1}^{1}(M)$ complementary to the coexact tensors. By the Helmholtz decomposition (which can be found by combining the Hodge-Morrey and Friedrich's decompositions, see [10]) we know that any $h \in \Lambda^{2}(M)$ can be uniquely written as the sum of a coexact form and a closed form with zero tangential component. We can identify antisymmetric tensors in $\tau_{1}^{1}(M)$ with elements of $\Lambda^{2}(M)$ through the metric, and so in fact for any $f \in \tau_{1}^{1}(M)$ we can uniquely decompose $f$ as

$$
f=f_{s}+(\alpha)^{\sharp}+(\delta \beta)^{\sharp}
$$

where $f_{s}=\left(f+f^{t}\right) / 2$ is the symmetric part of $f, \alpha \in \Lambda^{2}(M)$ is closed with tangential component zero, which means $i^{*} \alpha=0$, and $\beta \in \Lambda^{3}(M)$. According to theorem 3 we cannot expect to fully recover $(\delta \beta)^{\sharp}$ from the polarization data. However, we should be able to find at least the normal component of $f$ at the boundary since this is the part of a coexact tensor field $f=(\delta \beta)^{\sharp}$ that is determined by the values of $\beta$ on $\partial M$, which according to theorem 3 is exactly what determines the polarization data of $f$. 
Furthermore, we might expect to be able to recover $f$ fully on $\partial M$ when the coexact part of $f,(\delta \beta)^{\sharp}$, is zero. The present result in the three dimensional case is that in fact the above conjectures are true. More precisely we have the following.

Theorem 4. Let $(M, g)$ be a CNT manifold of dimension 3, let $f \in \tau_{1}^{1}(M)$ be a smooth tensor field. Then $\mathbf{n} f$ is uniquely determined by the polarization data of $f$. Further, if

$$
f=f_{s}+(\alpha)^{\sharp}
$$

is the decomposition of $f$ described above (note that $f$ has no coexact component), then in any coordinates all derivatives of all components of $f_{s}$ and $(\alpha)^{\sharp}$ on $\partial M$ are uniquely determined by the polarization data of $f$.

\section{Main Identity}

The proofs of our results are based upon an integral identity along the geodesics of $(M, g)$. The technique is inspired by the work of Stefanov and Uhlmann [11], and Uhlmann and Wang [12] on the boundary rigidity problem, and the identity comes from that used by Novikov and Sharafutdinov in [2] integrated along geodesics. We will write down a somewhat more general identity than is actually required to prove theorems 1 and 4 because it requires little extra work.

Let $(M, g)$ be as above and $f_{1}, f_{2} \in \tau_{1}^{1}(M)$. Let $U_{1}$ and $U_{2}$ be the solutions to $(1.2)$ corresponding to $f_{1}$ and $f_{2}$ respectively. Now fix any unit speed geodesic $\gamma$ between points $x, y \in \partial M$. In particular, assume that $\gamma(0)=x$, and $\gamma(l)=y$. Also, let us define $\dot{\gamma}(t)=\xi(t) \in T_{\gamma(t)} M$. If we choose any vectors $\eta \in T_{x}^{\mathbb{C}} M$ and $\zeta \in T_{y}^{\mathbb{C}} M$ then by equation (5.8) of $[2]$ we have

$$
\begin{aligned}
\left.\frac{\partial}{\partial s}\left\langle\left(U_{2}(\xi(s))^{-1} U_{1}(\xi(s))-E\right)\right) \mathcal{I}_{0, s}^{\gamma} \eta, \mathcal{I}_{l, s}^{\gamma} \zeta\right\rangle_{g(\gamma(s))}= \\
\left\langle U_{2}^{-1}(\xi(s))\left[P_{\xi(s)}\left(f_{1}-f_{2}\right)\right](\gamma(s)) U_{1}(\xi(s)) \mathcal{I}_{0, s}^{\gamma} \eta, \mathcal{I}_{l, s}^{\gamma} \zeta\right\rangle_{g(\gamma(s))}
\end{aligned}
$$

where $E$ is the identity. If we integrate this last equation from 0 to $l$ and use (5.9) from [2] we obtain

$$
\begin{aligned}
&\left.\left\langle\left(U_{2}(\xi(l))^{-1} U_{1}(\xi(l))-E\right)\right) \mathcal{I}_{0, l}^{\gamma} \eta, \zeta\right\rangle_{g(y)} \\
&=\int_{0}^{l}\left\langle U_{2}^{-1}\left[P_{\xi(s)}\left(f_{1}-f_{2}\right)\right](\gamma(s)) U_{1} \mathcal{I}_{0, s}^{\gamma} \eta, \mathcal{I}_{l, s}^{\gamma} \zeta\right\rangle_{g(\gamma(s))} \mathrm{d} s .
\end{aligned}
$$

Formula (3.1) is our main identity. We now take note of two special cases of this formula one of which we will use to prove theorems 1 and 4, and one of which is of general interest for the inverse problem. For our purposes in this work we will need the special case of (3.1) in which $f_{1}=f$ and $f_{2}=0$. For convenience we also denote $U_{1}=U$. It is clear that in this case $U_{2}=E$. We thus have

$$
\begin{aligned}
&\langle(U(\xi(l))-E)\left.\mathcal{I}_{0, l}^{\gamma} \eta, \zeta\right\rangle_{g(y)}= \\
& \int_{0}^{l}\left\langle\left[P_{\xi(s)} f\right](\gamma(s)) U(\xi(s)) \mathcal{I}_{0, s}^{\gamma} \eta, \mathcal{I}_{l, s}^{\gamma} \zeta\right\rangle_{g(\gamma(s))} \mathrm{d} s .
\end{aligned}
$$


Considering the entire inverse problem, that of determining $f$ on all of $M$ from its polarization data, it also seems useful to look at the special case of (3.1) when $f_{1}$ and $f_{2}$ give the same polarization data. Since $\xi(l) \in \partial_{+} \Omega M$, in this case the left hand side of (3.1) is zero. Thus, when the polarization data is the same we have

$$
0=\int_{0}^{l}\left\langle U_{2}^{-1}\left[P_{\xi(s)}\left(f_{1}-f_{2}\right)\right](\gamma(s)) U_{1} \mathcal{I}_{0, s}^{\gamma} \eta, \mathcal{I}_{l, s}^{\gamma} \zeta\right\rangle_{g(\gamma(s))} \mathrm{d} s .
$$

\section{Proof of Theorem 1}

Let $(M, g)$ and $f$ be as in the statement of the theorem, and let $U$ be the corresponding solution of (1.2). Our task is to determine all the derivatives, in some set of coordinates, of $f$ restricted to $\partial M$ from the polarization data $\left.U(x, \xi)\right|_{\partial_{+} \Omega M}$ of $f$ and the metric $g$. Take $x_{0} \in \partial M$. As a first step we will define the coordinate system near $x_{0}$ in which we will determine the derivatives. By the convexity of the boundary we can find a coordinate neighborhood $V$ centered at $x_{0}$ such that for every $x \in \partial M \cap V$ there is a unique geodesic contained in $V$ connecting $x_{0}$ and $x$. In these coordinates we now locally and smoothly extend both $f$ and the metric $g$ beyond $V \cap M$ to an open neighborhood of $x_{0}$. Having done this we now take normal coordinates centered at $x_{0}$ in the extended metric on a smaller neighborhood $U \subset V$. By rotating if necessary we can further assume that in these coordinates the outward pointing unit normal at $x_{0}$ is given by $-\partial_{x^{n}}$. It is in these normal coordinates that we will recover $f$ and its derivatives. Representing them as $\left(x^{\prime}, x^{n}\right)$ where $x^{\prime}=\left(x^{1}, \ldots, x^{n-1}\right)$, by convexity the boundary is given by a function $\phi\left(x^{\prime}\right)=x^{n}$ where $\phi(0)=0, D \phi(0)=0$, and $D^{2} \phi(0)>0$.

Now choose any two real vectors $\eta$ and $\zeta \in T_{x_{0}}^{\mathbb{C}} M$ and extend these to vector fields on $U$ by simply making them constant in the coordinates on $U$. Since the dimension of $\partial M$ is $\geq 3$, we may find a unit vector $\xi \in T_{x_{0}} \partial M$ that is perpendicular to both $\eta$ and $\zeta$. It is because of this step that we must require dimension $n \geq 4$, although we remark at this point that if either $\eta$ and $\zeta$ are parallel, or one of $\eta$ or $\zeta$ is normal to $\partial M$ we can still find such a $\xi$ in the three dimensional case. Now we consider the curve $c(\tau)$ in $\partial M$ given in the coordinates $\left(x^{\prime}, x^{n}\right)$ by (see figure 1 )

$$
c(\tau)=(\tau \xi, \phi(\tau \xi)) \quad, \quad \tau \geq 0 .
$$

For each $\tau$ in the domain of $c$, let $\gamma_{\tau}(t)$ be the unique geodesic contained in $V$ connecting $x_{0}$ and $c(\tau)$. Now, we apply (3.2) along the geodesic $\gamma_{\tau}(t)$ to obtain

$$
\begin{aligned}
\left\langle\left(U\left(\dot{\gamma}_{\tau}(t(\tau))\right)-E\right) \mathcal{I}_{0, t(\tau)}^{\gamma_{\tau}} \eta, \zeta\right\rangle_{g(c(\tau))} & = \\
& \int_{0}^{t(\tau)}\left\langle\left[P_{\dot{\gamma}_{\tau}(s)} f\right]\left(\gamma_{\tau}(s)\right) U\left(\dot{\gamma}_{\tau}(s)\right) \mathcal{I}_{0, s}^{\gamma_{\tau}} \eta, \mathcal{I}_{t(\tau), s}^{\gamma_{\tau}} \zeta\right\rangle_{g\left(\gamma_{\tau}(s)\right)} \mathrm{d} s
\end{aligned}
$$

where $t(\tau)$ is the distance from $x_{0}$ to $c(\tau)$. Since we assume we know the polarization data $\left.U\right|_{\partial_{+} M}$ and the metric $g$, the left side of this equation is entirely known. Let us call this known function $\psi(\tau)$. Then formally differentiating the last identity with respect 


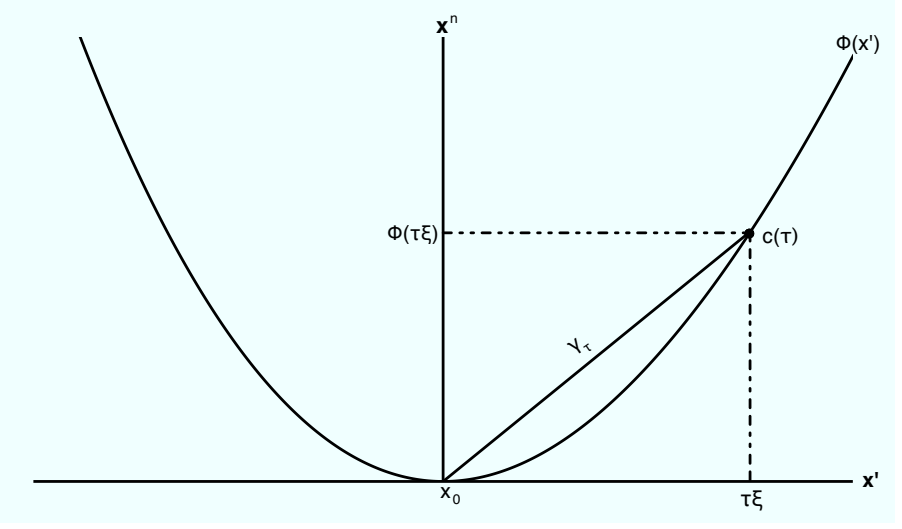

Figure 1. Boundary diagram

to $\tau$ we have

$$
\begin{aligned}
\psi^{\prime}(\tau)=t^{\prime}(\tau)\langle & {\left.\left[P_{\dot{\gamma}_{\tau}(t(\tau))} f\right] U \mathcal{I}_{0, t(\tau)}^{\gamma_{\tau}} \eta, \zeta\right\rangle_{g\left(\gamma_{\tau}(t)\right)} } \\
& +\int_{0}^{t(\tau)} \frac{\partial}{\partial \tau}\left\langle\left[P_{\dot{\gamma}_{\tau}(s)} f\right] U \mathcal{I}_{0, s}^{\gamma_{\tau}} \eta, \mathcal{I}_{t(\tau), s}^{\gamma_{\tau}} \zeta\right\rangle_{g\left(\gamma_{\tau}(s)\right)} \mathrm{d} s .
\end{aligned}
$$

Here and throughout this proof we suppress some of the dependences on $\gamma_{\tau}$ in order to save space. The next step will be to take the limit $\tau \rightarrow 0^{+}$, but to do this we need to justify the differentiation and study the behavior of all the functions involved as $\tau \rightarrow 0^{+}$. Note in particular that we must take care because the derivatives of $U(x, \xi)$ are generally unbounded near $\partial M$. To accomplish the analysis we will actually extend the various functions in (4.1) to smooth functions of $\tau$ in a neighborhood of 0 (note at the moment everything is only defined for $\tau>0$ ).

We first consider $t(\tau)$. Since we are using normal coordinates for $\tau>0$ we have

$$
t(\tau)=\sqrt{\|\tau \xi\|^{2}+\phi(\tau \xi)^{2}}=\tau \sqrt{1+\left(\frac{\phi(\tau \xi)}{\tau}\right)^{2}} .
$$

Since $\phi(0)=0$ this last formula gives a smooth function for $\tau$ such that $\tau \xi$ lies in the domain of $\phi$, and thus we use this formula to extend $t(\tau)$. For notational convenience we will write $\rho(\tau)=\phi(\tau \xi) / \tau$. As above we easily see that $\rho(\tau)$ is actually a smooth function, and by convexity of the boundary we also have $\rho(0)=D \phi(0) \cdot \xi=0$ and $\rho^{\prime}(0)=\left(\xi^{T} D^{2} \phi(0) \xi\right) / 2 \neq 0$. Now, calculating the derivative of $t(\tau)$ we obtain

$$
t^{\prime}(\tau)=\sqrt{1+\rho(\tau)^{2}}+\tau \frac{\rho(\tau) \rho^{\prime}(\tau)}{\sqrt{1+\rho(\tau)^{2}}} .
$$

Using this formula we see that

$$
\lim _{\tau \rightarrow 0^{+}} t^{\prime}(\tau)=1 .
$$

The analysis of $\gamma_{\tau}$ and $\dot{\gamma}_{\tau}$ also becomes quite clear because of the normal coordinates 
in which $\gamma_{\tau}$ is given by a straight line. For $\tau>0$

$$
\gamma_{\tau}(s)=\left(\frac{s \tau}{t(\tau)} \xi, \frac{s}{t(\tau)} \phi(\tau \xi)\right)=\left(s \frac{\xi}{\sqrt{1+\rho(\tau)^{2}}}, s \frac{\rho(\tau)}{\sqrt{1+\rho(\tau)^{2}}}\right)
$$

and

$$
\dot{\gamma}_{\tau}(s)=\left(\frac{\tau}{t(\tau)} \xi, \frac{\phi(\tau \xi)}{t(\tau)}\right)=\left(\frac{\xi}{\sqrt{1+\rho(\tau)^{2}}}, \frac{\rho(\tau)}{\sqrt{1+\rho(\tau)^{2}}}\right) .
$$

As above we now see that both of these formulas may be used to extend $\gamma_{\tau}(s)$ and $\dot{\gamma}_{\tau}(s)$ smoothly to the same domain as we have for $t(\tau)$. Further, we can calculate the limit

$$
\lim _{\tau \rightarrow 0^{+}} \dot{\gamma}_{\tau}(t(\tau))=(\xi, 0)
$$

Also note that since we extended $g$ to a neighborhood of $x_{0}$ and we are using normal coordinates centered at $x_{0}, \gamma_{\tau}$ is still a unit speed geodesic for $g$ even when $\tau \leq 0$.

There is no problem with $P_{\dot{\gamma}_{\tau}(s)} f$ since after we have extended $\dot{\gamma}_{\tau}$ smoothly for $\tau \leq 0$ this is evidently smooth with respect to $\tau$. The same is true of the metric $g\left(\gamma_{\tau}(s)\right)$ and the parallel translations. Thus the final term to analyze is $U\left(\dot{\gamma}_{\tau}(s)\right) \mathcal{I}_{0, s}^{\gamma_{\tau}} \eta$, however we see that this is a solution to the ODE

$$
\frac{D \omega}{d s}=\left(P_{\dot{\gamma}_{\tau}(s)} f\right)\left(\gamma_{\tau}(s)\right) \omega \quad, \quad \omega(0)=\eta
$$

where the right hand side depends smoothly on the parameter $\tau$. Therefore by proposition 6.2 in [13] $U\left(\dot{\gamma}_{\tau}(s)\right) \mathcal{I}_{0, s}^{\gamma_{\tau}} \eta$ is a smooth function of $\tau$ and $s$. Also, by continuity we easily see that as $\tau$ goes to $0^{+}, U\left(\dot{\gamma}_{\tau}(t(\tau))\right) \mathcal{I}_{0, t(\tau)}^{\gamma_{\tau}} \eta$ goes to $\eta$.

Having shown that all the functions in (4.1) are smooth, there is no problem differentiating with respect to $\tau$ under the integral, and we are now prepared to take the limit in (4.1). Indeed, letting $\tau \rightarrow 0^{+}$in that formula we have

$$
\psi^{\prime}(0)=\left\langle\left[P_{\xi} f\right] \eta, \zeta\right\rangle_{g\left(x_{0}\right)}=\left\langle f\left(x_{0}\right) \eta, \zeta\right\rangle_{g\left(x_{0}\right)} .
$$

The last equality in this equation follows because $\xi$ is perpendicular to both $\eta$ and $\zeta$. Thus we are able to recover $\left\langle f\left(x_{0}\right) \eta, \zeta\right\rangle_{g\left(x_{0}\right)}$ for any real vectors $\eta, \zeta \in T_{x_{0}}^{\mathbb{C}} M$, and so we can recover $f\left(x_{0}\right)$. This completes the task of proving that the values of $f$ on the boundary are completely determined by the polarization data.

Now we continue to recover the derivatives of $f$ as well. The argument will be by induction on the order of the derivatives, and the first inductive step will be to find all of the first order derivatives of $f$ at $x_{0}$. In order to clarify the method I will explicitly show how this initial step is accomplished. The method for the general step is essentially the same, but more complicated, and will also be explained below.

Since $f$ is already known on the boundary, all of its first order tangential derivatives are known, and so all that remains is to find $\partial_{x^{n}} f\left(x_{0}\right)$. This will be accomplished by taking two additional derivatives of (4.1). Throughout this argument we will refer to any function which is already known for all values of $\tau$ as $G(\tau)$, and any function which is known at $s=t(\tau)$ as $G(s, \tau)$. At this point the first term on the right hand side of (4.1) is $G(\tau)$ (ie. is a known function), and so after taking two additional derivatives it is 
still $G(\tau)$. Now let us consider the derivatives of the second, integral, term of (4.1). To begin this process we will partially evaluate the derivative contained in the integrand. All parts of this integrand are known except $f$, and so we separate out the term where the differentiation falls on $f$. Performing this partial expansion of the derivative using (4.2) yields

$$
\begin{gathered}
\frac{\partial}{\partial \tau}\left\langle\left[P_{\dot{\gamma}_{\tau}(s)} f\left(\gamma_{\tau}(s)\right)\right] U \mathcal{I}_{0, s}^{\gamma_{\tau}} \eta, \mathcal{I}_{t(\tau), s}^{\gamma_{\tau}} \zeta\right\rangle_{g\left(\gamma_{\tau}(s)\right)} \\
=\frac{s \rho^{\prime}(\tau)}{\sqrt{1+\rho(\tau)^{2}}}\left\langle\left[P_{\dot{\gamma}_{\tau}}\left(\partial_{x^{n}} f-\frac{\rho(\tau)}{1+\rho(\tau)^{2}}\left(D_{x^{\prime}} f \cdot \xi+\rho(\tau) \partial_{x^{n}} f\right)\right)\right] U \mathcal{I}_{0, s}^{\gamma_{\tau}} \eta, \mathcal{I}_{t(\tau), s}^{\gamma_{\tau}} \zeta\right\rangle_{g} \\
\quad+G(s, \tau) .
\end{gathered}
$$

Here $D_{x^{\prime}} f$ is the component-wise gradient of $f$ with respect to the first $n-1$ variables. We use this formula and the fact that $t(\tau)=\tau \sqrt{1+\rho(\tau)^{2}}$ to partially expand the second derivative of the integral term from (4.1) as follows (note that $\rho(\tau)=\mathcal{O}(\tau)$ )

$$
\begin{aligned}
\frac{\partial^{2}}{(\partial \tau)^{2}}\left(\int_{0}^{t(\tau)} \frac{\partial}{\partial \tau}\left\langle\left[P_{\dot{\gamma}_{\tau}(s)} f\right] U \mathcal{I}_{0, s}^{\gamma_{\tau}} \eta, \mathcal{I}_{t(\tau), s}^{\gamma_{\tau}} \zeta\right\rangle_{g\left(\gamma_{\tau}(s)\right)} \mathrm{d} s\right) \\
=t^{\prime}(\tau) \rho^{\prime}(\tau)\left\langle\left[P_{\dot{\gamma}_{\tau}(t(\tau))} \partial_{x^{n}} f\right] U \mathcal{I}_{0, t}^{\gamma_{\tau}} \eta, \zeta\right\rangle_{g\left(\gamma_{\tau}(t(\tau))\right)}+G(\tau)+\mathcal{O}(\tau) .
\end{aligned}
$$

Using this last equation we can take two additional derivatives with respect to $\tau$ in (4.1) and obtain

$$
\psi^{\prime \prime \prime}(\tau)=t^{\prime}(\tau) \rho^{\prime}(\tau)\left\langle\left[P_{\dot{\gamma}_{\tau}(t(\tau))} \partial_{x^{n}} f\right] U \mathcal{I}_{0, t}^{\gamma_{\tau}} \eta, \zeta\right\rangle_{g\left(\gamma_{\tau}(t(\tau))\right)}+G(\tau)+\mathcal{O}(\tau) .
$$

Then, taking $\tau \rightarrow 0^{+}$and solving we have

$$
\frac{\psi^{\prime \prime \prime}(0)-G(0)}{\rho^{\prime}(0)}=\left\langle\partial_{x^{n}} f\left(x_{0}\right) \eta, \zeta\right\rangle_{g\left(x_{0}\right)} .
$$

Thus we have recovered all first order derivatives of $f$ at $x_{0}$, and repeating this argument at any other point in the boundary we may recover all first order derivatives of $f$ on the entire boundary. Now we indicate how the general induction step is accomplished.

Let us suppose that we already know $\partial^{\beta} f(x)$ for all multi-indices $\beta$ with $|\beta| \leq m-1$ and all $x \in \partial M$. Then by differentiating in the boundary we also know $D_{x^{\prime}} \partial^{\beta} f\left(x_{0}\right)$ where $D_{x^{\prime}}$ is, as above, the gradient with respect to the first $n-1$ variables. Thus we know all of the $m^{\text {th }}$ order derivatives of $f$ at $x_{0}$ except for $\partial_{x^{n}}^{m} f\left(x_{0}\right)$. Now, let us differentiate (4.1) $2 m$ times. I claim that after doing this we obtain

$\psi^{(2 m+1)}(\tau)=m ! t^{\prime}(\tau) \rho^{\prime}(\tau)^{m}\left\langle\left[P_{\dot{\gamma}_{\tau}(t)} \partial_{x^{n}}^{m} f\right] U \mathcal{I}_{0, t}^{\gamma_{\tau}} \eta, \zeta\right\rangle_{g\left(\gamma_{\tau}(t)\right)}+G(\tau)+\mathcal{O}(\tau)$.

In order to show that (4.3) is correct, let us examine closely what happens when the right side of (4.1) is differentiated $2 m$ times. The first term on the right hand side is known on the boundary, and so differentiating with respect to $\tau$ results in a new known function. From the integral term we obtain first another integral term

$$
\int_{0}^{t(\tau)} \frac{\partial^{2 m}}{\partial \tau^{2 m}}\left\langle\left[P_{\left.\dot{\gamma}_{\tau}(s)\right)} f\right] U \mathcal{I}_{0, s}^{\gamma_{\tau}} \eta, \mathcal{I}_{t(\tau), s}^{\gamma_{\tau}} \zeta\right\rangle_{g\left(\gamma_{\tau}(s)\right)} \mathrm{d} s=\mathcal{O}(\tau),
$$


and then a plethora of terms in which one of the derivatives is applied to the $\tau$ in the upper limit of the integral. These terms can generally be grouped into three types. The first of these types occurs when fewer than $m$ derivatives are applied to $f$ while it is still under the integral sign. In this case, applying the fundamental theorem of calculus to evaluate the derivative with respect to the limit results in a known function by the induction hypothesis. The second type of term occurs when greater than $m$ derivatives are applied to $f$ while it is still under the integral sign. In this case we note that by (4.2), when $f$ is differentiated under the integral sign $k$ times the resulting integrand is $\mathcal{O}\left(s^{k}\right)$, and so after applying the fundamental theorem of calculus the term is $\mathcal{O}\left(\tau^{k}\right)$. If $k>m$, then after taking the remaining derivatives the term is still necessarily $\mathcal{O}(\tau)$. Finally, we have the terms where $f$ is differentiated exactly $m$ times while under the integral sign. As indicated above, after differentiating with respect to the limit of the integral this results in a term which is $\mathcal{O}\left(\tau^{m}\right)$, and therefore the only remaining term that is not $\mathcal{O}(\tau)$ is exactly the term where the remaining $m$ derivatives are applied to the $\tau^{m}$ factor. The factor in this term involving $f$ is

$$
t^{\prime}(\tau) \frac{m !}{\tau^{m}}\left(\left.\frac{\partial^{m}}{\partial \tau^{m}} f\left(\gamma_{\tau}(s)\right)\right|_{s=t(\tau)} .\right.
$$

Using the induction hypothesis that all $m^{\text {th }}$ and lower order derivatives of $f$ except $\partial_{x^{n}}^{m} f$ are known we can rewrite this as

$$
m ! t^{\prime}(\tau) \rho^{\prime}(\tau)^{m}\left(1-\frac{\rho(\tau)}{1+\rho(\tau)^{2}}\right)^{m} \partial_{x^{n}}^{m} f(\gamma(t(\tau)))+G(\tau) .
$$

Since $\rho(\tau)=\mathcal{O}(\tau)$ this can be written as

$$
m ! t^{\prime}(\tau) \rho^{\prime}(\tau)^{m} \partial_{x^{n}}^{m} f(\gamma(t(\tau)))+G(\tau)+\mathcal{O}(\tau) .
$$

Using this last expression and the preceding discussion we see that (4.3) is correct, and so after taking the limit $\tau \rightarrow 0^{+}$we may solve and obtain

$$
\frac{\psi^{(2 m+1)}(0)-G(0)}{\rho^{\prime}(0)^{m} m !}=\left\langle\partial_{x^{n}}^{m} f\left(x_{0}\right) \eta, \zeta\right\rangle_{g\left(x_{0}\right)} .
$$

This completes the induction step, and therefore completes the proof of theorem 1 .

\section{Proof of Theorem 4}

Let $(M, g)$ and $f$ be as in the statement of theorem 4. Proceeding exactly as above in the proof of theorem 1 we are able, using the same type of coordinates as above centered at $x_{0} \in \partial M$, to recover

$$
\left\langle f\left(x_{0}\right) \eta, \eta\right\rangle_{g\left(x_{0}\right)} \quad \text { and } \quad\left\langle f\left(x_{0}\right) \zeta, \nu\right\rangle_{g\left(x_{0}\right)}
$$

for any real vectors $\eta \in T_{x_{0}}^{\mathbb{C}}(M)$, and $\zeta \in T_{x_{0}}^{\mathbb{C}}(\partial M)$. Here $\nu$ is the outward pointing unit normal vector at $x_{0}$. The second of the quantities in (5.1) gives $\mathbf{n} f$, and so the first claim of theorem 4 is proven. Next we apply the same induction argument as in the proof of theorem 1 taking advantage of the special form (2.1) of $f$ to recover the derivatives. 
Indeed, assume that the coexact part of $f$ is zero and so $f$ has the form

$$
f=f_{s}+(\alpha)^{\#}
$$

where $f_{s}$ and $\alpha$ are as in the statment of the theorem. Then the first part of (5.1) gives $\mathbf{t} f_{s}$, and by assumption $\mathbf{t} \alpha=0$. Thus we actually recover $f$ fully on the boundary. This is sufficient for the base case of the induction. Suppose now that we know $\partial^{\beta} f$ on $\partial M$ for all mutli-indices $\beta$ with $|\beta| \leq m-1$. Then proceeding as in the proof of theorem 1 we obtain

$$
\left\langle\partial^{\beta} f\left(x_{0}\right) \eta, \eta\right\rangle_{g\left(x_{0}\right)} \quad \text { and } \quad\left\langle\partial^{\beta} f\left(x_{0}\right) \zeta, \nu\right\rangle_{g\left(x_{0}\right)}
$$

for all $\beta$ with $|\beta| \leq m$ and for any real vectors $\eta \in T_{x_{0}}^{\mathbb{C}}(M)$, and $\zeta \in T_{x_{0}}^{\mathbb{C}}(\partial M)$. The first part of (5.2) gives all derivatives up to order $m$ of $f_{s}$ and so the second part gives all derivatives up to order $m$ of $\mathbf{n} \alpha=\alpha-i^{*} \alpha$ (recall that $\alpha$ is the closed part of $f$ ). To recover the derivatives of $\mathbf{t} \alpha=i^{*} \alpha$ we will use the fact that $\alpha$ is closed.

To do this, suppose that $\alpha=\alpha_{12} d x^{1} \wedge d x^{2}+\alpha_{13} d x^{1} \wedge d x^{3}+\alpha_{23} d x^{2} \wedge d x^{3}$ in the coordinates defined above. Then at $x_{0}$ we have $\mathbf{t} \alpha=\alpha_{12} d x^{1} \wedge d x^{2}$ and $\mathbf{n} \alpha=\alpha_{13} d x^{1} \wedge d x^{3}+\alpha_{23} d x^{2} \wedge d x^{3}$. Since $\alpha$ is closed we have

$$
\partial_{x^{3}} \alpha_{12}-\partial_{x^{2}} \alpha_{13}+\partial_{x^{1}} \alpha_{23}=0 \quad \Rightarrow \quad \partial_{x^{3}} \alpha_{12}=\partial_{x^{2}} \alpha_{13}-\partial_{x^{1}} \alpha_{23} .
$$

Therefore we can find all the derivatives of $\mathbf{t} \alpha$ at $x_{0}$ up to order $m$ from the derivatives of just $\mathbf{n} \alpha$. This completes the proof by induction.

\section{References}

[1] Yu. A. Kravtsov and Yu. I. Orlov. Geometrical Optics of Inhomogeneous Media. Springer Series on Wave Phenomena, 6. Springer-Verlag, Berlin, 1990. 312 pp.

[2] R. Novikov and V. A. Sharafutdinov. On the problem of polarization tomography. I. Inverse Probl., 23(3):1229-1257, 2007.

[3] V.A. Sharafutdinov. Integral geometry of tensor fields. Inverse and Ill-posed Problems Series. Utrecht: VSP., 1994.

[4] H. Aben. Integrated Photoelasticity. McGraw-Hill, 1979. 203 pp.

[5] L. Ainola and H. Aben. Principal formulas of integrated photoelasticity in terms of characteristic parameters. J. Opt. Soc. A, 22:1181-1186, 2005.

[6] L. Ainola and H. Aben. Factorization of the polarization transformation matrix in integrated photoelasticity. J. Opt. Soc. A, 24(11):3397-3402, 2007.

[7] H Hammer and W. R. B. Lionheart. Reconstruction of spatially inhomogeneous dielectric tensors through optical tomography. J. Opt. Soc. Am. A, 22(2):250-255, 2005.

[8] H Hammer and B. Lionheart. Application of Sharafutdinov's ray transform in Integrated photoelasticity. J. Elasticity, 75(3):229-246, 2005.

[9] L. B. Vertgeim. Integral geometry with matrix weight and one nonlinear problem in matrix reconstruction. Soviet Math. Dokl., 44(1):132-135, 1992.

[10] G. Schwarz. Hodge decomposition - A Method for Solving Boundary Value Problems. Lecture Notes in Mathematics, 1607. Springer-Verlag, Berlin, 1995. 155 p.

[11] P. Stefanov and G. Uhlmann. Rigidity for metrics with the same lengths of geodesics. Math. Res. Lett., 5(1-2):83-96, 1998.

[12] G. Uhlmann and J.-N. Wang. Boundary determination of a Riemannian metric by the localized boundary distance function. Adv. Appl. Math., 31(2):379-387, 2003.

[13] M. Taylor. Partial Differential Equations I. New York : Springer, 1996. 